Check for updates

Cite this: J. Mater. Chem. A, 2019, 7, 2921

DOI: $10.1039 /$ c9ta90027f

www.rsc.org/MaterialsA

\section{Expression of concern: Mesoporous amorphous FeOF nanococoons for high-rate and long-life rechargeable sodium-ion batteries}

Sam Keltie

Expression of concern for 'Mesoporous amorphous FeOF nanococoons for high-rate and long-life rechargeable sodium-ion batteries' by Shi Yan Fu et al., J. Mater. Chem. A, 2015, 3, 16716-16727.

The following article 'Mesoporous amorphous FeOF nanococoons for high-rate and long-life rechargeable sodium-ion batteries' by Shi Yan Fu, Yuan Zhi Li, Wei Chu, Yi Mei Yang, Dong Ge Tong and Qing Le Zeng has been published in Journal of Materials Chemistry A. The article reports the synthesis of mesoporous amorphous FeOF nanococoons for sodium-ion batteries.

Journal of Materials Chemistry $A$ is publishing this expression of concern in order to alert our readers that we are presently unable to confirm the accuracy of the data reported in Fig. 1a, of this Journal of Materials Chemistry A paper and Fig. S5a, S8, S9i, S9k, S12c-d, S13e, S14a, S26a, and S27a-b of the ESI.

An investigation is underway, and this notice will be updated when a final outcome is reached.

Sam Keltie

$17^{\text {th }}$ January 2019

Executive Editor, Journal of Materials Chemistry A 